\title{
DISTRIBUTION OF VULPIA SPECIES (POACEAE) IN POLAND
}

\author{
LUdWIK FREY $^{1}$, BEATA PASZKO ${ }^{1}$, PAWEŁ KWIATKOWSKI ${ }^{2}$ \\ ${ }^{1} \mathrm{~W}$. Szafer Institute of Botany, Polish Academy of Sciences \\ Lubicz 46, 31-512 Kraków, Poland \\ e-mail: L.Frey@ib-pan.krakow.pl \\ e-mail: paszko@ib-pan.krakow.pl \\ 2 Department of Botany and Plant Physiology \\ University of Agricultural Sciences \\ Cybulskiego 32, 50-205 Wrocław, Poland \\ e-mail: pkwiat@ozi.ar.wroc.pl
}

(Received: April 1, 2003. Accepted: July 18, 2003)

\begin{abstract}
The distribution of four species of the genus Vulpia [V. myuros (L.) C.C. Gmel., V. bromoides (L.) S.F. Gray, $V$. ciliata Dumort. and V. geniculata (L.) Link] reported in Poland has been studied. Currently, V. myuros and especially $V$. bromoides are very rare species, and their greatest concentration can be found only in the Lower Silesia region. The number of their localities decreased after 1950 and it seems resonable to include both species in the „red list” of threatened plants in Poland: V. myuros in the EN category, V. bromoides in the CR category. $V$. ciliata and $V$. geniculata are very rare ephemerophytes and their localities not confirmed during ca 60 years are of historical interest only.
\end{abstract}

KEY WORDS: Vulpia, distribution, taxonomy, hybrids, Lower Silesia, Poland.

\section{INTRODUCTION}

The species of the Vulpia genus are distributed mainly in temperate and subtropical regions of the northern hemisphere. They are also introduced to the southern hemisphere (e.g. Australia), although perhaps several endemic species occur in South America. Over the whole area of their distribution the species of Vulpia grow on dry, open places (Clayton and Renvoize 1986; Watson and Dallwitz 1992).

The purpose of the present paper is the verification of distribution of four Vulpia species reported hitherto in Poland: V. myuros (L.) C.C. Gmel., V. bromoides (L.) S.F. Gray, V. ciliata Dumort. and V. geniculata (L.) Link and their threat. The verification is based on revised herbarium materials, data from the reliable literature and unpublished data.

\section{TAXONOMY, KARYOLOGY AND HYBRIDS}

In its widest historical sense the genus Vulpia contains ca 30 species divided into several groups treated at various times either as distinct genera or as subgenera or sections. Currently, the species of Vulpia are placed in 5 or $6 \mathrm{sec}-$ tions. The studied species belong to two sections: VulpiaV. myuros C.C. Gmel., V. bromoides (L.) S.F. Gray, V. ci- liata Dumort. and Loretia - V. geniculata (L.) Link (Cotton and Stace 1976, 1977; Stace 1981; Clayton and Renvoize 1986; Conert et al. 1998).

Vulpia myuros and $V$. bromoides were originally placed in the genus Festuca L., and to-day certain authors still follow this practice. However, both species differentiate distictly from representatives of Festuca in respect of habit, florets and lemmas (Cotton and Stace 1977; Auquier and Stace 1980). Most close to the Festuca species (from the section Ovinae) is Vulpia geniculata (Bulińska-Radomska and Lester 1986). The morphological differences between the four studied species are fairly significant (Conert et al. 1998; Rutkowski 1998).

In the genus there are three ploidy levels (diploid to hexaploid). The most frequent chromosome number is $2 n=$ 14 (e.g. $V$. bromoides, $V$. geniculata). The numbers $2 \mathrm{n}=28$ (V. ciliata) and 42 (V. myuros) have been also found (Cotton and Stace 1976, 1977; Goldblatt 1985; Goldblatt and Johnson 1994). In Poland the number $2 \mathrm{n}=42$ for $V$. myuros has been established in plants from the southern part of the country (Mizianty et al. 1981).

Vulpia is closely related to Festuca L., and both genera easily hybridize each other. Natural, rare intergeneric hybrids have been found (Stace and Cotton 1974; Ainscough et al. 1986). No natural interspecific Vulpia hybrids are known. 


\section{DISTRIBUTION OF STUDIED SPECIES}

\section{General distribution}

The area of greatest genetic diversity of the genus is the western Mediterranean region, especially the Italian and Iberian Peninsulas and adjacent parts of North Africa. The most widely distributed are species of the Vulpia section, which occur throughout most of Europe, North Africa and western Asia. The representatives of other sections have a rather restricted area of distribution (Cotton and Stace 1976).

Vulpia myuros is widespread in western, central and southern Europe (reaching as far north as Ireland and central England), and reaching as far east as central Asia. In America, Australia, eastern Asia and southern Africa it is distributed as an introduced plant (Cotton and Stace 1976; Stace and Cotton 1980). In the opinion of Zając and Zając (2001b) this species belongs to Mediterranean-Irano-Turanian connective element. The maps of its general distribution are presented by Hultén (1962), Meusel et al. (1965) and Conert et al. (1998).

Vulpia bromoides occurs in northern Scotland and in southern Sweden, where it is a threatened species, but the centre of its distribution is the north-western part of the European continent. It is very occasionally found in Russia and western Asia. The species is well naturalized in North and South America and in Australia (Cotton and Stace 1976; Stace and Cotton 1980; Ingelög et al. 1993). Its geographical range is presented by Meusel et al. (1965) and Hultén and Fries (1986)

Vulpia ciliata occurs mainly in western and southern Europe, northwards to $53^{\circ}$ in eastern England (Stace and Auquier 1978; Stace and Cotton 1980; Lambinon et al. 1992).

Vulpia geniculata is distributed in the western and central parts of the Mediterranean area ranging from Portugal to southern France (Cotton and Stace 1976; Stace and Cotton 1980). In central, northern and eastern Europe and in the British Isles it is sporadically introduced mainly with wool and bird-seeds (Ryves et al. 1996; Conert et al. 1998).

\section{Distribution in Poland}

In the 19th and at the begining of the 20th century Vulpia myuros has been reported mainly in western Poland, from the lowland and foothills of the Sudeten (rarerly Carpathians) as a fairly frequent species (Berdau 1859; Rostafiński 1872; Abromeit et al. 1940; Ascherson and Graebner 1898-1899; Schube1903-1929; Zapałowicz 1906; Szafer 1919; Szafer et al. 1924). The majority of localities were found before 1950. In the second half of the 20th century, however, the number of localities of that species markedly decreased (Table 1, Fig. 1) (Szafer et al. 1953; Mowszowicz 1978; Falkowski 1982; Rutkowski 1998, 2002; Mirek

TABLE 1. Occurrence of Vulpia myuros in Poland before and after 1950 (number of localities)

\begin{tabular}{lcc}
\hline Source of data & Year & up to 1950 \\
\hline Herbarium & 12 & 25 \\
Literature & 90 & 22 \\
Unpublished & - & 13 \\
\hline
\end{tabular}

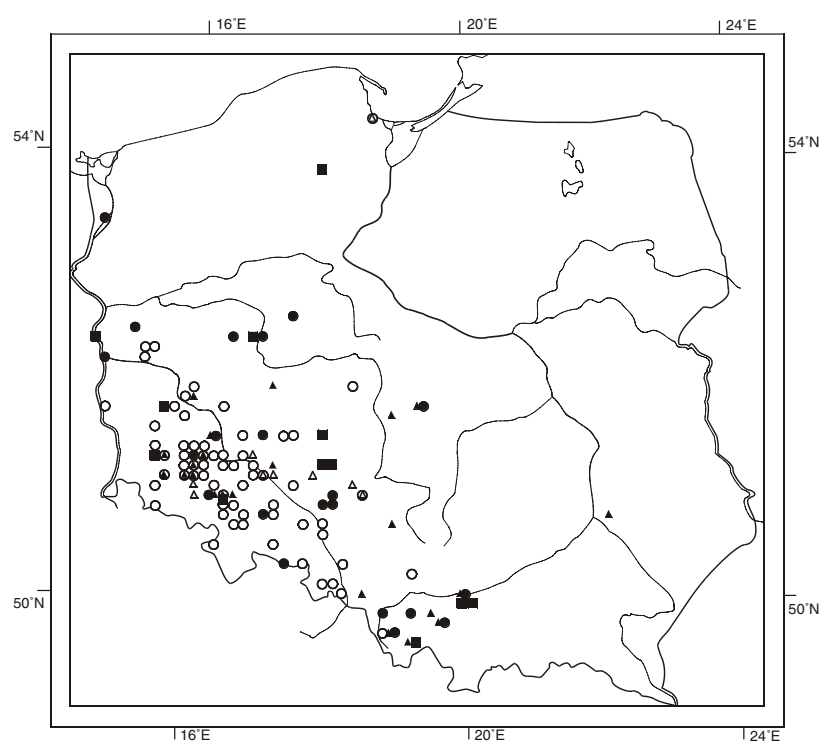

Fig. 1. Past and present distribution of Vulpia myuros in Poland: triangles - herbarium materials, circles - literature records, squares - unpublished data (white - localities before 1950, black - localities after 1950).

and Piękoś-Mirkowa 2002). It should be emphasized that in the last 12 years (1991-2002) V. myuros was found in 18 localities only. According to Rutkowski (2002) it is an apophyte, but in some areas it should be treated rather as a kenophyte.

Vulpia bromoides is a rarer species than the former one. Its localities have been recorded most frequently before 1950 .

TABLE 2. Occurrence of Vulpia bromoides in Poland before and after 1950 (number of localities).

\begin{tabular}{lcc}
\hline Source of data & up to 1950 & 1951-2002 \\
\hline Herbarium & - & 5 \\
Literature & 29 & 5 \\
Unpublished & - & 2 \\
\hline
\end{tabular}

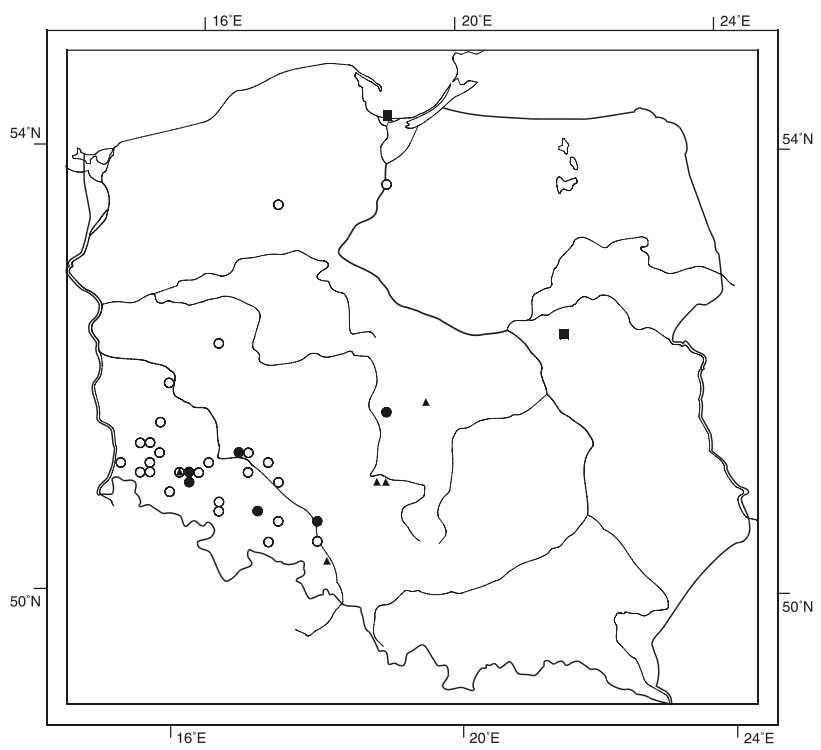

Fig. 2. Past and present distribution of Vulpia bromoides in Poland: triangles - herbarium materials, circles - literature records, squares - unpublished data (white - localities before 1950, black - localities after 1950). 
In the second half of the 20th century the number of localities of the species has apparently diminished. Between 1951 and 2002 it was reported in a few sites only and in 12 last years (1991-2002) - merely in three (Szafer et al. 1924, 1953; Mowszowicz 1978; Falkowski 1982; Rutkowski 1998) (Table 2, Fig. 2). It is considered to be an ephemerophyte (Rostański and Sowa 1986-1987; Frey and Rutkowski 2002; Korniak 2002; Mirek et al. 2002; Rutkowski 2002).

Vulpia myuros and V. bromoides attain in Poland local eastern limits of their distribution (Ingelög et al. 1993; $\mathbf{Z u -}$ kowski and Jackowiak 1995; Piękoś-Mirkowa and Mirek 2002). They are character species of the Vicio lathyroidisPotentillion argenteae Brzeg in Brzeg et M. Wojt. 1996 alliance and of Filagini-Vulpietum, the pioneer plant community, where $V$. myuros is the dominant species, while occurrence of $V$. bromoides is only ephemeral (Brzeg and Wojterska 1996; Matuszkiewicz 2001).

Vulpia ciliata and $V$. geniculata are extremely rare ephemerophytes. V. ciliata was found in Wrocław by Schalow (1932 - city street) as a new taxon to Silesia, and one year later was confirmed by Meyer (1933 - railway station). Its occurrence in Szczecin (Scheuermann 1956 - railway station, leg. G. Wangrin, 1942), was not confirmed by Ćwiklinski (1970). On the occurrence of $V$. geniculata only one mention from a single locality in Gubin (1929) was reported by Lademann (1937).

\section{CONCLUSIONS}

(1) The greatest concentration of Vulpia myuros and $V$. bromoides can be found in the Lower Silesia region (Dolny Śląsk).

(2) Nowadays Vulpia myuros is a rare species, although in the first half of 20th century it was recorded in western and south-western Poland in fairly numerous localities. However, the majority of them has not been confirmed during the second half of 20th century.

(3) The occurrence of Vulpia myuros has been recently confirmed in 3 localities only, and between 1991 and 2002 it was found in 15 new localities.

(4) Thus the map of its distribution in Poland (Zając and Zając 2001a) was modified by the present authors (Fig. 1).

(5) Vulpia bromoides has been recently confirmed only in one locality, and between 1991 and 2002 it was found solely in three new localities. A map of its distribution in Poland is published in the present paper for the first time (Fig. 2).

(6) Vulpia myuros and V. bromoides are character species of Filagini-Vulpietum, a very rare plant community in western Poland (Ziemia Lubuska, Bory Dolnośląskie and Wzgórza Trzebnickie regions). This community has been included in the group of endangered natural communities with unknown dynamic tendencies, and usually with insufficient documentation (Brzeg and Wojterska 1996).

(7) Thus, it seems reasonable to consider both species as stable elements of the Polish flora, though recently they have being decreased in abundance, and for this reason the present authors propose to include them in the "red list" of threatened plants in Poland: Vulpia myuros in the EN category, and $V$. bromoides in the CR category (it is worthy of notice, that in the Sudeten Mts V. myuros has been included in the EN category, while $V$. bromoides in the VU category - Fabiszewski and Kwiatkowski 2002).
(8) Vulpia ciliata and V. geniculata are ephemerophytes and their occurrence in Poland has been hitherto noted only incidentally and extremely rarely. The localities of both species have not been confirmed during ca 60 years, therefore are of historical interest only.

\section{LOCALITIES OF VULPIA MYUROS}

\section{Herbarium materials}

AE: 29 - Bolesławiec, 1970, leg. Wójcikiewicz (WRSL) $51^{\circ} 16^{\prime} \mathrm{N} / 14^{\circ} 35^{\prime} \mathrm{E}$; 2002, leg. P. Kwiatkowski (private herbarium); 49 - Lwówek Śląski, 1993, leg. P. Kwiatkowski (private herbarium) $51^{\circ} 07^{\prime} \mathrm{N} / 15^{\circ} 35^{\prime} \mathrm{E}$

BD: 62 - Grochowice, 1980, leg. K. Browicki (WRSL) $51^{\circ} 47^{\prime} \mathrm{N} / 16^{\circ} 0^{\prime} \mathrm{E}$

BE: 04 - Podgórze, 1961, leg. Z. Gtowacki (WRSL) $51^{\circ} 31^{\prime} \mathrm{N} / 16^{\circ} 16^{\prime} \mathrm{E}$; Toszowice, 1961, leg. Z. Głowacki, (WRSL), 1972, leg. E. Koziot (BIL) 51 ${ }^{\circ} 28^{\prime}$ N $/ 16^{\circ} 18^{\prime} \mathrm{E} ; 23$ - Dobrzejów, 1965, leg. K. Rostański (WRSL) 51 ${ }^{\circ} 15^{\prime} \mathrm{N} /$ $16^{\circ} 11^{\prime}$ E; 28 - Oborniki Śląskie, 17.06.1867, leg. $R$. Uechtritz (WRSL); $51^{\circ} 18^{\prime}$ N/16 ${ }^{\circ} 55^{\prime}$ E; 32 - Legnica, 1998, leg. P. Kwiatkowski (private herbarium) $51^{\circ} 13^{\prime} \mathrm{N} /$ $16^{\circ} 10^{\prime}$ E; 41 - Kozia Góra, 375 m a.s.1., 1995, leg. $P$. Kwiatkowski (private herbarium) $51^{\circ} 05^{\prime} \mathrm{N} / 15^{\circ} 57^{\prime} \mathrm{E} ; 42$ Słup, 2002, leg. P. Kwiatkowski (private herbarium) 51 $06^{\prime} \mathrm{N} / 16^{\circ} 06^{\prime} \mathrm{E} ; 49$ - Rędzin (Wrocław-Psie Pole), 1881, leg. Uechtritz (WRSL) $51^{\circ} 10^{\prime} \mathrm{N} / 17^{\circ} 05^{\prime} \mathrm{E} ; \mathbf{5 2}-$ Wielisławka $369 \mathrm{~m}$ a.s.1. near Świerzawa, 16.06.1894, leg. Pinkwart (WRSL), 1993, leg. P. Kwiatkowski (private herbarium) $51^{\circ} 01^{\prime} \mathrm{N} / 15^{\circ} 54^{\prime} \mathrm{E}$; 62 - Wolbromek, 25.06.1874, leg.? (WRSL) $50^{\circ} 56^{\prime} \mathrm{N} / 16^{\circ} 04^{\prime} \mathrm{E} ; 64$ - Strzegom, 1973, leg. E. Koziot (KTU) 50 $58^{\prime} \mathrm{N} / 16^{\circ} 21^{\prime}$ E; 65 - Łażany, 1965, leg. J. Madalski, (JM) 5057' N/16³0’ E; $66-$ Strzeblów, 1966, leg. W. Stojanowska, (WRSL) 5054' N/ $16^{\circ} 43^{\prime} \mathrm{E}$

CD: 50 - Lipówka, 1995, leg. A. Czarna (POZ) 5158’ N/ $17^{\circ} 09^{\prime} \mathrm{E}$

CE: 30 - Borowa, 1963, leg. Z. Głowacki (WRSL) $51^{\circ} 11^{\prime} \mathrm{N} / 17^{\circ} 17^{\prime} \mathrm{E} ; 40$ - Szczodre, 1859, leg. Uechtritz (WRSL) $51^{\circ} 12^{\prime} \mathrm{N} / 17^{\circ} 11^{\prime} \mathrm{E} ; 44$ - Kowalowice, 1887, leg.?, (WRSL) $51^{\circ} 08^{\prime} \mathrm{N} / 17^{\circ} 46^{\prime} \mathrm{E}$; between Namysłów and Byczyna, 1888, leg.?, (WRSL) $51^{\circ} 06^{\prime} \mathrm{N} / 17^{\circ} 58^{\prime} \mathrm{E} ; 58$ Pawłowice Gorzowskie,?, leg. H. Zuschke (WRSL) 51 ${ }^{\circ} 01^{\prime} \mathrm{N} /$ $18^{\circ} 24^{\prime} \mathrm{E} ; 69$ - Biskupice?, leg. H. Zuschke (WRSL) 50 57' N/18 29' E, Skrońsko, leg. H. Zuschke (WRSL) 5059’ N/ $18^{\circ} 27^{\prime} \mathrm{E}$

CF: 69 - Boguszowice, 1959, leg. J. Janota (LOD) $50^{\circ} 02^{\prime} \mathrm{N} / 18^{\circ} 34^{\prime} \mathrm{E}$; Niedobczyce, 1959, leg. J. Janota (LOD) $50^{\circ} 03^{\prime} \mathrm{N} / 18^{\circ} 28^{\prime} \mathrm{E}$

DA: 80 - Gdańsk-Westerplatte, 1843, leg. Klinsmann (TRN) $54^{\circ} 25^{\prime} \mathrm{N} / 18^{\circ} 40^{\prime} \mathrm{E}$

DD: 75 - Łódź-Rokicie, 1965, leg. R. Sowa (LOD) $51^{\circ} 44^{\prime}$ N/19²6' E; 82 - Szadek, 1967, leg. R. Sowa (LOD) $51^{\circ} 42^{\prime} \mathrm{N} / 18^{\circ} 59^{\prime} \mathrm{E}$

DE: 92 - Herby Nowe, 1978, leg. K. Ptak (KTU) 5040’ N/ $18^{\circ} 53^{\prime} \mathrm{E}$

DF: 69 - Kraków-Zakrzówek, 2001, leg. J. Guzik (KRAM) 5001' N/1954' E; 86 - Gorzeń Dolny, 1992, leg. E. Markiewicz (KTU) 4952’ N/19³0’ E; 97 - Skawce, 1999 , leg. W. Bartoszek (private herbarium) $49^{\circ} 48^{\prime} \mathrm{N} /$ $19^{\circ} 35^{\prime}$ E; Dąbrówka, 1997, leg. W. Bartoszek (private herbarium) $49^{\circ} 48^{\prime} \mathrm{N} / 19^{\circ} 37^{\prime} \mathrm{E}$ 
DG: 02 - Brenna, 1935, leg. J. Gałuszka; 1974, leg. K. Rostański (KTU) 4943' N/18 54' E; 14 - Wieprz, 1987, leg. M. Skolarz (KTU) 49³8' N/19¹0’ E

FE: 84 - Zaklików, 1970, leg. D. Fijałkowski, (LBL) $50^{\circ} 45^{\prime} \mathrm{N} / 22^{\circ} 06^{\prime} \mathrm{E}$

\section{Literature}

AB: 83 - Szczecin (Scheuermann 1956) 5326' N/14³4' E AC: 96 - Sulęcin (Ćwikliński 1995) $52^{\circ} 26^{\prime} \mathrm{N} / 15^{\circ} 07^{\prime} \mathrm{E}$

AD: 17 - Toporów (Decker) 52 $16^{\prime} \mathrm{N} / 15^{\circ} 16^{\prime} \mathrm{E} ; 18$ Mostki (Decker (1911) 52 ${ }^{\circ} 16^{\prime} \mathrm{N} / 15^{\circ} 24^{\prime} \mathrm{E}$; Ołobok (Decker 1911) $52^{\circ} 13^{\prime} \mathrm{N} / 1^{\circ} 26^{\prime} \mathrm{E} ; 23$ - Rąpice (Ćwikliński 1995) $52^{\circ} 06^{\prime} \mathrm{N} / 14^{\circ} 44^{\prime} \mathrm{E} ; 27$ - Zawisze (Decker 1911) $52^{\circ} 09^{\prime} \mathrm{N} / 15^{\circ} 19^{\prime} \mathrm{E} ; 73$ - Brożek (Decker 1911) $51^{\circ} 43^{\prime} \mathrm{N} /$

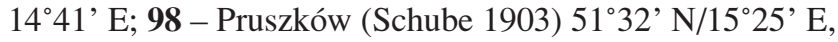
Małomice (Schube 1903) 51 $33^{\circ}$ N $/ 15^{\circ} 27^{\prime} \mathrm{E}$

AE: 18 - Kliczków (Schube 1903) 51²0’ N/15²6’ E; Osiecznica (Schube 1903) 51 ${ }^{\circ} 20^{\prime}$ N $/ 15^{\circ} 25^{\prime}$ E; 29 - Bolesławiec (Schube 1914) 51 '16' N/15³4' E; Godnów (Schube 1903) $51^{\circ} 16^{\prime} \mathrm{N} / 15^{\circ} 38^{\prime} \mathrm{E}$; 49 - Lwówek Śląski (Schube 1903) $51^{\circ} 07^{\prime} \mathrm{N} / 15^{\circ} 35^{\prime}$ E; 58 - Popielówek (Schube 1903) $50^{\circ} 59^{\prime} \mathrm{N} / 15^{\circ} 31^{\prime} \mathrm{E} ; \mathbf{7 8}$ - Cieplice Śląskie-Zdrój (Schube 1903) $50^{\circ} 52^{\prime} \mathrm{N} / 15^{\circ} 35^{\prime} \mathrm{E}$

BD: 06 - Poznań-Górczyn, (Żukowski 1960) 52²2' N/16 53'E; 09 - Poznań-Franowo, (Żukowski 1960) $52^{\circ} 22^{\prime} \mathrm{N} / 17^{\circ} 01^{\prime} \mathrm{E} ; \mathbf{5 2}$ - between Krępina and Lubiatów (Schube 1903) 5156’ N/1558' E; Święte (Schube 1903) $51^{\circ} 55^{\prime} \mathrm{N} / 15^{\circ} 57^{\prime} \mathrm{E} ; 61$ - Borowiec (Schube 1903) $51^{\circ} 48^{\prime} \mathrm{N} /$ $15^{\circ} 53^{\prime}$ E, Lipiny (Gruhl 1929) 51 $52^{\prime} \mathrm{N} / 15^{\circ} 49^{\prime} \mathrm{E} ; \mathbf{7 0}_{-}$ between Drwalowice and Lasocin (Schube 1903) $51^{\circ} 43^{\prime} \mathrm{N} /$ $15^{\circ} 41^{\prime} \mathrm{E}$; 75 - Laskowa (Schube 1903) 51 ${ }^{\circ} 46^{\prime}$ N N/16 31' E, Radosław-Strupina (Schube 1903) 51 ${ }^{\circ} 45^{\prime}$ N/16 $26^{\circ}$ E; 81 Mieszków (Schube 1903) 51³8’ N/1552’ E

BE: 04 - Chełmek Wołowski (Głowacki 1962) 51 ${ }^{\circ} 27^{\prime} \mathrm{N} /$ $16^{\circ} 21^{\prime}$ E; 07 - Białawy Małe (Schube 1903) 51 29' N/ $16^{\circ} 42^{\prime}$ E; 09 - „Stawy Milickie” near Ruda Sułowska (Anioł-Kwiatkowska et al. 1995) $51^{\circ} 32^{\prime} \mathrm{N} / 17^{\circ} 06^{\prime} \mathrm{E} ; 11$ Rokitki (Schube 1903) 51 21' N/1554' E, Duninów (Schube 1903) $51^{\circ} 24^{\prime}$ N/15 $57^{\prime}$ E; 12 - Trzebnice (Schube 1907) $51^{\circ} 23^{\prime} \mathrm{N} / 16^{\circ} 01^{\prime} \mathrm{E} ; \mathbf{1 3}$ - Lubin (Schube 1903) $51^{\circ} 24^{\prime} \mathrm{N} /$ $16^{\circ} 12^{\prime}$ E; 21 - Chojnów (Schube 1903) $51^{\circ} 17^{\prime}$ N $/ 15^{\circ} 56^{\prime} \mathrm{E}$; Czernikowice (Schube 1903) $51^{\circ} 18^{\prime}$ N $/ 15^{\circ} 54^{\prime}$ E; Jaroszów-

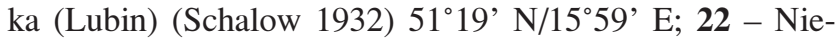
dźwiedzice (Schube 1903) 51 ${ }^{\circ} 17^{\prime} \mathrm{N} / 16^{\circ} 02^{\prime} \mathrm{E}$; Raszówka (Schube 1903) $51^{\circ} 19^{\prime} \mathrm{N} / 16^{\circ} 07^{\prime} \mathrm{E}$; Grzymalin, (Anioł and Pender 1971) $51^{\circ} 17^{\prime} \mathrm{N} / 16^{\circ} 06^{\prime} \mathrm{E} ; 23$ - Bieniowice (Schube 1903) $51^{\circ} 16^{\prime} \mathrm{N} / 16^{\circ} 11^{\prime} \mathrm{E}$; Rzeszotary (Schube 1903) $51^{\circ} 16^{\prime} \mathrm{N} / 16^{\circ} 09^{\prime} \mathrm{E}$; Dobrzejów (Schube 1903) $51^{\circ} 15^{\prime} \mathrm{N} /$ $16^{\circ} 11^{\prime} \mathrm{E} ; 24$ - Szczytniki (Schube 1903) $51^{\circ} 17^{\prime} \mathrm{N} / 16^{\circ} 17^{\prime} \mathrm{E}$; Lisowice (Schube 1903) 52 $17^{\prime}$ N/16 ${ }^{\circ} 22^{\prime}$ E; 25 - Lubiąż (Schube 1903) $51^{\circ} 16^{\prime} \mathrm{N} / 16^{\circ} 28^{\prime}$ E; 27 - Rościsławice (Schube 1903) $51^{\circ} 18^{\prime} \mathrm{N} / 16^{\circ} 50^{\prime} \mathrm{E} ; 31$ - Podolany (Schube 1903) $51^{\circ} 11^{\prime} \mathrm{N} / 15^{\circ} 58^{\prime} \mathrm{E} ; 32$ - Wilczyce (Schube 1903) $51^{\circ} 10^{\prime} \mathrm{N} / 16^{\circ} 04^{\prime} \mathrm{E} ; 33$ - Kunice (Schube 1903) 51 $1^{\circ} 14^{\prime} \mathrm{N} /$ $16^{\circ} 15^{\prime}$ E; Pątnów Legnicki (Schube 1903) 5114' N/16¹3' E; 35 - Prawików (Schube 1903) 51 ${ }^{\circ} 15^{\prime} \mathrm{N} / 16^{\circ} 30^{\prime} \mathrm{E}$; 36 - between Brodno and Szczepanów (Schube 1915) 51 ${ }^{\circ} 12^{\prime} \mathrm{N} /$ $16^{\circ} 36^{\prime}$ E; 38 - Wrocław-Świniary (Schube 1903) $51^{\circ} 12^{\prime}$ N/ $16^{\circ} 58^{\prime}$ E; 41 - Wilków (Schube 1905) 51 06’ N/1556' E; 42 - Krotoszyce (Schube 1903) $51^{\circ} 09^{\prime}$ N/1603' E; Górzec

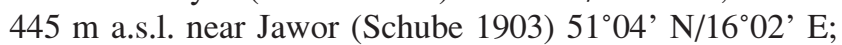

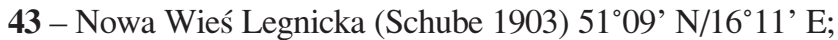

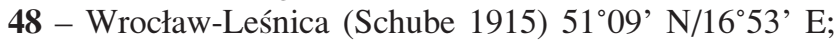

49 - Wrocław-Popowice (Schube 1903) $51^{\circ} 08^{\prime} \mathrm{N} / 17^{\circ} 00^{\prime} \mathrm{E}$; Wrocław (Meyer 1932, 1936) $51^{\circ} 07^{\prime} \mathrm{N} / 17^{\circ} 02^{\prime} \mathrm{E} ; \mathbf{5 4}$ - Targoszyn (Schube 1903) 51 01' N/16²18' E; 57 - Nowa Wieś Kącka (Schalow 1932) 51 $01^{\circ}$ N/16 44' E; Krobielowice (Schube 1903) $51^{\circ} 00^{\prime} \mathrm{N} / 16^{\circ} 48^{\prime} \mathrm{E}$; 64 - Strzegom (Stojanowska 1973) $50^{\circ} 58^{\prime} \mathrm{N} / 16^{\circ} 21^{\prime} \mathrm{E}$; 65 - Pyszczyn (Schube 1903) $50^{\circ} 59^{\prime} \mathrm{N} / 16^{\circ} 32^{\prime} \mathrm{E} ; \mathbf{7 5}$ - Kraszowice (Schube 1903, 1929) 5059' N/16²9' E; 76 - Strzeblów (Schube 1903) $50^{\circ} 54^{\prime}$ N $/ 16^{\circ} 43^{\prime}$ E; 85 - Krzyżowa (Schube 1903) 5048' N/ $16^{\circ} 32^{\prime}$ E; 87 - Gola Dzierżoniowska (Schube 1903) 5044' N/ $16^{\circ} 47^{\prime}$ E; 89 - Strzelin (Stojanowska 1973) $50^{\circ} 47^{\prime} \mathrm{N} /$ $17^{\circ} 04^{\prime}$ E; 96 - between Nowa Wieś Niemczańska and Piława Górna (Schube 1925) 5041' N/1644' E; 97 - Piekiełko near Niemcza (Schalow 1931) 50 43' N/16 50 ' E, Niemcza-Jasin (Schalow 1931) 5043' N/16 50 ' E

BF: 14 - Radków (Schube 1903) 50³0' N/16²4' E

CC: 82 - Imiołki (Balcerkiewicz et al. 1997) 52 33' N/ $17^{\circ} 23^{\prime} \mathrm{E}$

CD: 58 - Pawłowice Gorzowskie (Schube 1903) 5101' N/ $18^{\circ} 24^{\prime} \mathrm{E}$;

CE: 01 - Duchowo (Schube 1916) $51^{\circ} 31^{\prime} \mathrm{N} / 17^{\circ} 19^{\prime} \mathrm{E}$, Milicz-Kobiałka (Schube 1916) $51^{\circ} 32^{\prime} \mathrm{N} / 17^{\circ} 17^{\prime}$ E, Krośnice (Schube 1929) $51^{\circ} 28^{\prime} \mathrm{N} / 1^{\circ} 22^{\prime} \mathrm{E} ; \mathbf{0 2}$ - Sławoszowice (Schube 1915) $51^{\circ} 32^{\prime}$ N/17 $17^{\circ}$ ' E; 52 - Kolonia Miłocice (Schube 1914) $51^{\circ} 12^{\prime}$ N $/ 17^{\circ} 38^{\prime}$ E; 66 - Markotów Mały (Sendek 1966) 50 59' N/18 06’ E; 69 - Biskupice (Schube 1903) $50^{\circ} 57^{\prime}$ N $/ 18^{\circ} 29^{\prime}$ E, Skrońsko (Schube 1903) 5059’ N/

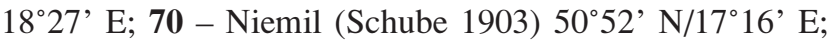
75 - Murów (Michalak 1965) 5051’ N/17 $56^{\circ}$ E; 76 - Bukowo (Sendek 1966, 1970) 5054' N/18 06’ E; 80 - Krajno

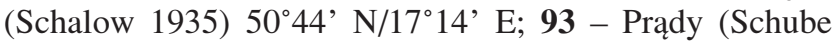
1903) $50^{\circ} 39^{\prime} \mathrm{N} / 17^{\circ} 43^{\prime}$ E; 95 - Osowiec Śląski (Schube 1903) $50^{\circ} 44^{\prime} \mathrm{N} / 17^{\circ} 59^{\prime} \mathrm{E}$

CF: 05 - Szczepanowice (Schube 1903) 50³9’ N/1754' E; 10 - between Goświnowice and Głębinów (Schube 1903) $50^{\circ} 28^{\prime} \mathrm{N} / 17^{\circ} 16^{\prime} \mathrm{E} ; 31$ - Głuchołazy (Schube 1903) $50^{\circ} 19^{\prime} \mathrm{N} / 17^{\circ} 23^{\prime} \mathrm{E}$; Jarnołtówek (Szotkowski 1971) 50¹7' N/ $17^{\circ} 25^{\prime} \mathrm{E}$; Biała Głuchołaska (Krawiecowa et al. 1963, after Schube 1902) $50^{\circ} 18^{\prime} \mathrm{N} / 17^{\circ} 22^{\prime} \mathrm{E} ; 33$ - Prudnik (Schalow 1933) $50^{\circ} 19^{\prime} \mathrm{N} / 17^{\circ} 36^{\prime}$ E; 37 - Kędzierzyn (Schalow 1931) $50^{\circ} 21^{\prime}$ N/18¹2' E; 55 - Raków Głubczycki (Schube 1903) $50^{\circ} 07^{\prime} \mathrm{N} / 18^{\circ} 01^{\prime} \mathrm{E} ; 56$ - Dobrosławice (Schube 1903) $50^{\circ} 12^{\prime} \mathrm{N} / 18^{\circ} 02^{\prime} \mathrm{E}$; Maciowakrze (Schube 1903) $50^{\circ} 11^{\prime} \mathrm{N} /$ $18^{\circ} 02^{\prime}$ E; 67 - Racibórz (Schube 1929) 5005’ N/18 $14^{\circ}$ ' E

DA: 80 - Gdańsk (Abromeit et al. 1940) $54^{\circ} 22^{\prime} \mathrm{N} /$ $18^{\circ} 36^{\prime} \mathrm{E}$

DD: 75 - Łódź-Rokicie (Sowa 1968, 1969, 1974) 5144' $\mathrm{N} / 19^{\circ} 26^{\prime} \mathrm{E}$

DF: 44 - near Dabrowa (Berdau 1859) 50¹3' N/19 $13^{\prime}$ E; 69 - Kraków-Łobzów (Kornaś et al. 1959) 5004' N/ $19^{\circ} 54^{\prime}$ E; 81 - Ochaby (Zając 1989) 49 $51^{\circ}$ N N/18 46' E; 84 - Kobiernice Górne (Kotońska 1991) 4951' N/19¹3' E; 97 - Skawce (Mizianty et al. 1981) $49^{\circ} 48^{\prime} \mathrm{N} / 19^{\circ} 35^{\prime} \mathrm{E}$

DG: 01 - Ustroń (Schube 1903) 4943' N/18 49' E; 02 Brenna-Leśnica (Celiński et al. 1976) 49 41' N/18 54' E; Brenna GRN (Celiński et al. 1976) 4943’ N/18 $58^{\prime}$ E

\section{Unpublished}

AD: 02 - Świecko, E. Ćwikliński, 1988, 52²18' N/14³5' E; 79 - Sokołów, E. Kuźniewski, unpbl. 1966 - ATPOL $51^{\circ} 44^{\prime} \mathrm{N} / 15^{\circ} 39^{\prime} \mathrm{E}$

AE: 28 - Bolesławice, K. Pender, 2002 - personal communication $51^{\circ} 17^{\prime} \mathrm{N} / 15^{\circ} 33^{\prime} \mathrm{E}$ 
BD: 08 - Poznań, B. Jackowiak, 1986 - ATPOL 52²4’ N/ $16^{\circ} 55^{\prime} \mathrm{E}$

BE: 65/75 - Wiśniowa, K. Pender, 2000 - personal communication $50^{\circ} 53^{\prime} \mathrm{N} / 16^{\circ} 29^{\prime} \mathrm{E}$

CB: 35 - Przytarnia, I. Kosiński, 1988 - ATPOL 535ํ' N/ $17^{\circ} 52^{\prime} \mathrm{E}$

CE: 05 - Antonin, J. Borysiak et al., 1993 - ATPOL $51^{\circ} 31^{\prime}$ N/17 $52^{\circ}$ E; 35 - Feliksów, A. Wawrzyniak, 1979 ATPOL $51^{\circ} 12^{\prime} \mathrm{N} / 17^{\circ} 57^{\prime} \mathrm{E}$; 36 - Laski, A. Wawrzyniak, 1979 - ATPOL $51^{\circ} 12^{\prime} \mathrm{N} / 18^{\circ} 02^{\prime} \mathrm{E}$

DF: 79 - Libertów 260, D. Tumidajowicz, 1965 - ATPOL $49^{\circ} 58^{\prime} \mathrm{N} / 19^{\circ} 53^{\prime} \mathrm{E}$

DG: 14 - Grojec Mt. near Żywiec, K. Nowak, 1997 personal communication $49^{\circ} 38^{\prime} \mathrm{N} / 19^{\circ} 10^{\prime} \mathrm{E}$

EF: 70 - Pawlikowice, D. Tumidajowicz, 1965 - ATPOL 49 $57^{\prime}$ N/20 04' E, Podlesie, D. Tumidajowicz, 1965 ATPOL $49^{\circ} 57^{\prime} \mathrm{N} / 19^{\circ} 58^{\prime} \mathrm{E}$

\section{LOCALITIES OF VULPIA BROMOIDES}

\section{Herbarium materials}

BE: 41 - Wilcza Góra 378 m a.s.1. near Złotoryja, 1991, leg. P. Kwiatkowski (private herbarium) $51^{\circ} 06^{\prime} \mathrm{N} / 17^{\circ} 55^{\prime} \mathrm{E}$

CF: 36 - Większyce, 1965, leg. T. Bereta (KRAM) $50^{\circ} 20^{\prime} \mathrm{N} / 18^{\circ} 06^{\prime} \mathrm{E}$

DD: 76 - Łódź-Rokicie, 1965, leg. R. Sowa (LOD) $51^{\circ} 44^{\prime} \mathrm{N} / 19^{\circ} 26^{\prime} \mathrm{E}$,

DE: 51 - Bobrowniki, Pajęczno, 1967, leg. R. Sowa (LOD) $51^{\circ} 05^{\prime} \mathrm{N} / 18^{\circ} 44^{\prime} \mathrm{E} ; \mathbf{5 2}$ - Raciszyn, 1967, leg. R. So$w a(\mathrm{LOD}) 51^{\circ} 06^{\prime} \mathrm{N} / 18^{\circ} 52^{\prime} \mathrm{E}$

\section{Literature}

AD: 99 - Dziećmiarowice (Schube 1917) 51³3’ N/ $15^{\circ} 35^{\prime} \mathrm{E}$

AE: 17 - Parowa (Schube 1903) 51 ${ }^{\circ} 22^{\prime}$ N $/ 15^{\circ} 17^{\prime}$ E; 18 Krępnica (Schube 1903) $51^{\circ} 20^{\prime}$ N/15 $29^{\circ}$ E; 29 - Bolesławiec (Schube 1906) 51 16' N/15³4' E, Godnów (Schube 1903) $51^{\circ} 16^{\prime} \mathrm{N} / 15^{\circ} 34^{\prime} \mathrm{E} ; 35$ - Zgorzelec (Schube 1903) $51^{\circ} 09^{\prime} \mathrm{N} / 15^{\circ} 01^{\prime} \mathrm{E} ; 38$ - Kraszowice (Schube 1903) $51^{\circ} 13^{\prime} \mathrm{N} /$ $15^{\circ} 31^{\prime} \mathrm{E} ; 47$ - Niwnice (Schube 1903) $51^{\circ} 08^{\prime} \mathrm{N} / 15^{\circ} 23^{\prime} \mathrm{E}$; 48 - Gradówek (Schube 1903) $51^{\circ} 07^{\prime} \mathrm{N} / 15^{\circ} 29^{\prime} \mathrm{E}$

BD: 15 - Lisowice (Schube 1903) 52 $17^{\prime}$ N/16 $22^{\prime}$ E; 50 - Pyrnik (Schube 1903) 51 $56^{\prime} \mathrm{N} / 15^{\circ} 46^{\prime} \mathrm{E}$

BE: 27 - Brzeg Dolny (Głowacki 1973, 1975) 51 ${ }^{\circ} 16^{\prime} \mathrm{N} /$ $16^{\circ} 43^{\prime}$ E; 28 - Oborniki Śląskie (Schube 1903) $51^{\circ} 18^{\prime} \mathrm{N} /$ $16^{\circ} 55^{\prime}$ E; 34 - Goślinów (Schube 1903), Jaśkowice (Schube 1903) $51^{\circ} 13^{\prime} \mathrm{N} / 16^{\circ} 18^{\prime}$ E; 41 - Sępów (Schube 1903) $51^{\circ} 06^{\prime} \mathrm{N} / 15^{\circ} 53^{\prime} \mathrm{E}$, Wilcza Góra $378 \mathrm{~m}$ a.s.l. near Złotoryja (Schube 1903) 51 $06^{\prime}$ N $/ 17^{\circ} 55^{\prime}$ E; 42 - Kopista (Widoma), $264 \mathrm{~m}$ a.s.1. near Jawor (Kwiatkowski 2000) $51^{\circ} 05^{\prime} \mathrm{N} /$ $16^{\circ} 06^{\prime}$ E; 43 - Nowa Wieś Legnicka (Schube 1903) 51 ${ }^{\circ} 09^{\prime}$ N/ $16^{\circ} 11^{\prime}$ E; 48 - Wrocław (Schube 1903, Meyer 1936) $51^{\circ} 07^{\prime} \mathrm{N} / 17^{\circ} 02^{\prime}$ E; 52 - Winna Góra (Winnica), 275 $\mathrm{m}$ a.s.1. near Piotrowice (Kwiatkowski 2001) $51^{\circ} 04^{\prime} \mathrm{N} /$ $16^{\circ} 07^{\prime}$ E; 60 - Wzgórze Kościuszki, 412 m a.s.l. near Jelenia Góra 5054' N/1544' E (Schube 1903); 75 - Pszenno (Schube 1925) 50 51' N/16 32' E, Marcinowice (Schube 1926) $50^{\circ} 53^{\prime}$ N $/ 16^{\circ} 35^{\prime}$ E; 85 - Lubachów (Schube 1909) $50^{\circ} 46^{\prime} \mathrm{N} / 16^{\circ} 26^{\prime} \mathrm{E} ; 89$ - Strzelin (Stojanowska 1973) $50^{\circ} 47^{\prime} \mathrm{N} / 17^{\circ} 04^{\prime} \mathrm{E}$

CB: 71 - Myśligoszcz (Abromeit et al. 1940) 53³5’ N/ $17^{\circ} 19^{\prime} \mathrm{E}$
CE: 30 - Szczodre (Schube 1903) $51^{\circ} 12^{\prime} \mathrm{N} / 17^{\circ} 11^{\prime} \mathrm{E}$; $\mathbf{5 1}$ - Kopalina (Schube 1912) $51^{\circ} 02^{\prime}$ N $/ 17^{\circ} 25^{\prime}$ E; 91 - Wilczyce (Schalow 1932) 5050' N/16 59' E; 95 - Opole

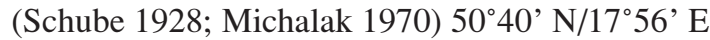

CF: 10 - Głębinów (Schube 1903) 50²8' N/17²16' E;

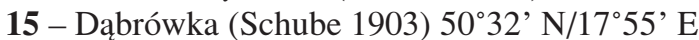

DB: 52 - Kwidzyn (Abromeit et al. 1940) 534' N/ $18^{\circ} 56^{\prime} \mathrm{E}$

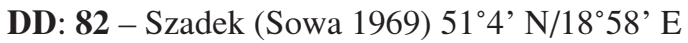

\section{Unpublished data}

DA: 82 - Mikoszewo, H. Skowron, 1977 - ATPOL $54^{\circ} 20^{\prime} \mathrm{N} / 18^{\circ} 57^{\prime} \mathrm{E}$

FD: 00 - Kąty-Flakowizna, Z. Głowacki, 1978 - ATPOL $52^{\circ} 21^{\prime} \mathrm{N} / 21^{\circ} 36^{\prime} \mathrm{E}$

\section{ACKNOWLEDGEMENTS}

The authors would like to thank Janusz Guzik M.Sc. for his help in localization of some localities and Prof. A. Zając for providing access to the ATPOL database. We are grateful to the Curators of herbaria for the loan of herbarium specimens and to all persons mentioned in the paper for information on the occurrence of the studied species.

\section{LITERATURE CITED}

ABROMEIT J., NEUHOFF W., STEFFEN H. 1940. Flora von Ost- und Westpreussen. 2, 5. Kommissionsverlag Gräfe und Unzer, Königsberg, pp. 877-1248.

AINSCOUGH M.M., BARKER C.M., STACE C.A. 1986. Natural hybrids between Festuca and species of Vulpia section Vulpia. Watsonia 16: 143-151.

ANIOŁ J., PENDER K. 1971. Materiały do flory Śląska. III. Flora okolic Legnicy. Acta Univ. Wratisl. 134, Pr. Bot. 12: 3-27. (in Polish with English summary)

ANIOŁ-KWIATKOWSKA J., BERDOWSKI W., KOŁA W., KWIATKOWSKI P., MACICKA T., PANEK E., PENDER K., WERETELNIK E., WILCZYŃSKA W. 1995. Charakterystyka botaniczna rezerwatu ornitologicznego „Stawy Milickie". Acta Univ. Wratisl. 1667, Pr. Bot. 62: 199-233. (in Polish with English summary)

ASCHERSON P., GRAEBNER P. 1898-1899. Flora des Nordostdeutschen Flachlandes. Verl. Von Gebrüder Borntraeger, Berlin, pp. 875.

AUQUIER P., STACE C.A. 1980. Variation in flowering behaviour in Vulpia (Poaceae). Pl. Syst. Evol. 136: 47-52.

BALCERKIEWICZ S., BRZEG A., RAKOWSKI W., GÓRSKI P. 1997. Airetum praecocis (Schwick. 1944) Krausch 1967 zbiorowisko przydroży i placów biwakowych na siedliskach piaszczystych. In: S. Wika, Roślinność obszarów piaszczystych. Uniw. Śląski i Zespół Jurajskich Parków Krajobrazowych Woj. Katowickiego, Katowice-Dąbrowa Górnicza, pp. 13-23. (in Polish with English and Russian summaries)

BERDAU F. 1859. Flora cracoviensis. Cracoviae. Typis C. R. Univ. Jagell., Cracovia, pp. 448.

BRZEG A., WOJTERSKA M. 1996. Przegląd systematyczny zbiorowisk roślinnych Wielkopolski wraz z oceną stopnia ich zagrożenia. Bad. Fizjogr. Pol. Zach. Ser. B - Botanika 45: 7-40. (in Polish with English summary)

BULIŃSKA-RADOMSKA Z., LESTER R.N. 1986. Relationship between seven species of Vulpia (Poaceae). Pl. Syst. Evol. 153: 7-12.

CELIŃSKI F., ROSTAŃSKI K., SENDEK A., WIKA S., CABAŁA S. 1976. Nowe stanowiska rzadkich roślin naczyniowych 
na Górnym Śląsku. Cz. III. Opol. Tow. Przyj. Nauk, Zesz. Przyr. 16: 15-31. (in Polish with English summary)

CLAYTON W.D., RENVOIZE S.A. 1986. Genera graminum. The grasses of the world. Her Majesty's Stationery Office, London, pp. 389.

CONERT H.J., JÄGER E.J., KADEREIT J.W., SCHULTZE-MOTEL W., WAGENITZ G., WEBER H.E. (eds) 1998. Gustav Hegi Illustrierte Flora von Mitteleuropa, 1 (3). Spermatophyta: Angiospermae: Monocotyledones 1 (2): Poaceae (Echte Gräser oder Süssgräser). 3. Aufl. Parey Buchverlag, Berlin, pp. 898.

COTTON R., STACE C.A. 1976. Taxonomy of the genus Vulpia (Gramineae). I. Chromosome numbers and geographical distribution of the Old World species. Genetica 46: 235-255.

COTTON R., STACE C.A. 1977. Morphological and anatomical variation of Vulpia (Gramineae). Bot. Notiser 130: 173-17.

ĆWIKLIŃSKI E. 1970. Flora synantropijna Szczecina. Monogr. Bot. 33: 1-103.

ĆWIKLIŃSKI E. 1995. Stanowiska rzadkich gatunków we florze województw gorzowskiego, szczecińskiego i północnej części zielonogórskiego. Zesz. Nauk. Wyż. Szk. Roln.-Ped. w Siedlcach Ser. Nauki Przyr. 44: 15-19.

DECKER P. 1911. Beiträge zur Flora der südlichen Neumark und der östlichen Niederlausitz. Verh. Bot. Vereins Prov. Branderburg 53: 87-269.

FABISZEWSKI J., KWIATKOWSKI P. 2002. Threatened vascular plants of the Sudeten Mountains. Acta Soc. Bot. Pol. 4: 339-350.

FALKOWSKI M. (ed.) 1982. Trawy polskie. PWR i L, Warszawa, pp. 565. (in Polish)

FREY L., RUTKOWSKI L. 2002. Wykaz gatunków (system według Clayton, Renvoize 1986, zmieniony). In: L. Frey (ed.), Polska księga traw. Instytut Botaniki im. W. Szafera, Kraków, pp. 87-95. (in Polish)

GŁOWACKI Z. 1962. Notatki florystyczne z powiatu wołowskiego. Fragm. Flor. Geobot. 8: 119-129. (in Polish with German summary)

GŁOWACKI Z. 1973. Notatki florystyczne z powiatu wołowskiego (Dolny Śląsk). Cz. III. Zesz. Przyr. Opol. Tow. Nauk 13: 41-47. (in Polish with German summary)

GŁOWACKI Z. 1975. Zbiorowiska murawowe zachodniej części Wzgórz Trzebnickich. Pr. Opol. Tow. Przyj. Nauk, Wydz. III - Nauk Przyr. PWN, Warszawa-Wrocław, pp. 102. (in Polish)

GOLDBLATT P. (ed.) 1985. Index to plant chromosome numbers 1982-1983. Monogr. Syst. Bot. Missouri Bot. Garden 13: 1-224.

GOLDBLATT P., JOHNSON D.E. (eds) 1994. Index to plant chromosome numbers 1990-1991. Monogr. Syst. Bot. Missouri Bot. Garden 51: 1-267.

GRUHL K. 1929. Tier und Pflanzenwelt der Kreises Grünberg. Druck u. Verlag W. Levy Sohn, Grünberg I. Schl., pp. 546.

HULTÉN E. 1962. The circumpolar plants. 1. Vascular cryptogams, conifers, monocotyledons. Almquist, Wiksell, Stockholm, pp. 275.

HULTÉN E., FRIES M. 1986. Atlas of North European vascular plants. North of the Tropic of Cancer. 1, 3. Koeltz Scientific Books, Königstein, pp. 498 and pp. 1172.

INGELÖG T., ANDERSSON R., TJERNBERG M. (eds) 1993. Red data book of the Baltic region. Part 1. List of threatened vascular plants and vertebrates. Swedish Threatened Species Unit, Uppsala \& Institute of Biology, Riga, pp. 95.

KORNAŚ J., LEŚNIEWSKA I., SKRZYWANEK A. 1959. Obserwacje nad florą linii kolejowych i dworców towarowych w Krakowie. Fragm. Flor. Geobot. 5: 199-216. (in Polish with German summary)

KORNIAK T. 2002. Trawy synantropijne. In: L. Frey (ed.), Polska księga traw. Instytut Botaniki im. W. Szafera, Kraków, pp. 277-300. (in Polish with English summary)

KOTOŃSKA B. 1991. Rośliny naczyniowe Beskidu Małego (polskie Karpaty Zachodnie). Zesz. Nauk. Uniw. Jagiell. 1020 Pr. Bot. 23: 7-199. (in Polish with English summary)
KRAWIECOWA A., KUCZYŃSKA I., GOŁOWIN S. 1963. Rośliny naczyniowe Gór Opawskich. Acta Univ. Wratisl. 14 Pr. Bot. 1: 3-142. (in Polish with English summary)

KWIATKOWSKI P. 2000. Notatki florystyczne z Gór Kaczawskich i ich Pogórza (Sudety Zachodnie). Fragm. Flor. Geobot. Polonica 7: 105-116. (in Polish with English summary)

KWIATKOWSKI P. 2001. Koniczyna kreskowana Trifolium striatum L. w Sudetach. Annales Silesiae 31: 53-58. (in Polish with English summary)

LADEMANN O. 1937. Adventivpflanzen der östlichen Niderlausitz. Verh. Bot. Ver. Prov. Brandenb. 77: 1-23.

LAMBINON J., DE LANGHE J.-E., DELVOSALLE L., DUVIGNEAUD J. 1992. Nouvelle flore de la Belgique, du GrandDuché de Luxembourg, du Nord de la France et des Régions voisines. Ed. 4. Edition du Patrimoine du Jardin botanique national de Belgique, Meise, pp. 1092.

MATUSZKIEWICZ W. 2001. Przewodnik do oznaczania zbiorowisk roślinnych Polski. Vademecum geobotanicum 3. Wydawnictwo Naukowe PWN, Warszawa, pp. 537. (in Polish)

MEUSEL H., JÄGER E., WEINERT E. 1965. Vergleichende Chorologie der Zentraleuropäischen Flora (Karten). G. Fischer, Jena, pp. 258.

MEYER K. 1932. Über den gegenwärtigen Stand der Bahnhofsfloristik in Schlesien. Jahres-Ber. Schl. Ges. Vaterl. Cultur 104: 76-91.

MEYER K. 1933. Neue schlesischen Adventivpflanzen. JahresBer. Schl. Ges. Vaterl. Cultur 105 (1932): 141-154.

MEYER K. 1936. Neue Fremdpflanzen Schlesiens. Jahres-Ber. Schl. Ges. Vaterl. Cultur 108: 81-89.

MICHALAK S. 1965. Materiały zielnikowe Muzeum Śląska Opolskiego. Opol. Tow. Przyj. Nauk, Zesz. Przyr. 5: 29-40. (in Polish with English summary)

MICHALAK S. 1970. Flora synantropijna miasta Opola. Opol. Roczn. Muz. 4: 1-181. (in Polish with German and English summaries)

MIREK M., PIĘKOŚ-MIRKOWA H. 2002. Trawy gór. In: L. Frey (ed.), Polska księga traw. Instytut Botaniki im. W. Szafera, Kraków, pp. 143-166. (in Polish with English summary)

MIREK M., PIĘKOŚ-MIRKOWA H., ZAJAC A., ZAJAC M. 2002. Flowering plants and Pteridophytes of Poland - a checklist. W. Szafer Institute of Botany, Polish Academy of Sciences, pp. 442.

MIZIANTY M., FREY L., MIREK Z. 1981. Contribution to the knowledge on the chromosome numbers of Polish vascular plants. Fragm. Flor. Geobot. 27: 19-29.

MOWSZOWICZ J. 1978. Conspectus florae Poloniae Medianae. Uniwersytet Łódzki, Łódź, pp. 395.

PIĘKOŚ-MIRKOWA H., MIREK Z. 2002. Zagrożenie i ochrona gatunkowa. In: L. Frey (ed.), Polska księga traw. Instytut Botaniki im. W. Szafera, Kraków, pp. 209-234. (in Polish with English summary)

ROSTAFIŃSKI J. 1873 [,,1872”]. Florae polonicae prodromus. Verh. k. k. zool.-bot. Ges. Wien.: 81-208.

ROSTAŃSKI K., SOWA R. 1986-1987. Alfabetyczny wykaz efemerofitów Polski. Fragm. Flor. Geobot. 31-32: 151-205. (in Polish with English summary)

RUTKOWSKI L. 1998. Klucz do oznaczania roślin naczyniowych Polski niżowej. Wydawnictwo Naukowe PWN, Warszawa, pp. 812. (in Polish)

RUTKOWSKI L. 2002. Trawy niżu. In: L. Frey (ed.), Polska księga traw. Instytut Botaniki im. W. Szafera, Kraków, pp. 167-185. (in Polish with English summary)

RYVES T.B., CLEMENT E.J., FOSTER M.C. 1996. Alien grasses of the British Isles. Botanical Society of the British Isles, London, pp. 181.

SCHALOW E. 1931. Ergebnisse der Durchforschung der Schlesischen Gefässpflanzenwelt im Jahre 1930. Jahres-Ber. Schl. Ges. Vaterl. Cultur 103: 116-132. 
SCHALOW E. 1932. Ergebnisse der Durchforschung der Schlesischen Gefässpflanzenwelt im Jahre 1931. Jahres-Ber. Schl. Ges. Vaterl. Cultur 104: 92-112.

SCHALOW E. 1933. Ergebnisse der Schlesischen Phanerogamenforschung im Jahre 1932. Jahres-Ber. Schl. Ges. Vaterl. Cultur 105: 154-173.

SCHALOW E. 1935. Ergebnisse der Durchforschung der Schlesischen Gefässpflanzenwelt im Jahre 1934. Jahres-Ber. Schles. Ges. Vaterl. Cultur 107: 55-71.

SCHEUERMANN R. 1956. Beitrag zur Adventivflora in Pommern. Decheniana 108 (1954/1955) 2: 169-196. Bonn.

SCHUBE T. 1903. Die Verbreitung der Gefässpflanzen in Schlesien, preussischen und šsterreichischen Anteils. Druck von R. Nischkowsky, Breslau, pp. iv +362 .

SCHUBE T. 1905. Ergebnisse der Durchforschung der schlesischen Gefässpflanzenwelt im Jahre 1904. Jahres-Ber. Schles. Ges. Vaterl. Cultur 82: 41-64.

SCHUBE T. 1906. Ergebnisse der Durchforschung der schlesischen Gefässpflanzenwelt im Jahre 1905. Jahres-Ber. Schles. Ges. Vaterl. Cultur 83: 75-95.

SCHUBE T. 1907. Ergebnisse der Durchforschung der schlesischen Gefässpflanzenwelt im Jahre 1906. Jahres-Ber. Schles. Ges. Vaterl. Cultur 84: 68-89.

SCHUBE T. 1909. Ergebnisse der Durchforschung der schlesischen Gefässpflanzenwelt im Jahre 1908. Jahres-Ber. Schles. Ges. Vaterl. Cultur 86: 48-66.

SCHUBE T. 1912. Ergebnisse der Durchforschung der schlesischen Gefässpflanzenwelt im Jahre 1911. Jahres-Ber. Schles. Ges. Vaterl. Cultur 89: 57-70.

SCHUBE T. 1914. Ergebnisse der Durchforschung der schlesischen Gefässpflanzenwelt im Jahre 1913. Jahres-Ber. Schles. Ges. Vaterl. Cultur 91: 133-155.

SCHUBE T. 1915. Ergebnisse der Durchforschung der schlesischen Gefässpflanzenwelt im Jahre 1914. Jahres-Ber. Schles. Ges. Vaterl. Cultur 92: 43-61.

SCHUBE T. 1916. Ergebnisse der Durchforschung der schlesischen Gefässpflanzenwelt im Jahre 1915. Jahres-Ber. Schles. Ges. Vaterl. Cultur 93: 35-45.

SCHUBE T. 1917. Ergebnisse der Durchforschung der schlesischen Gefässpflanzenwelt im Jahre 1916. Jahres-Ber. d. schles. Ges. f. vaterl. Cultur 94: 27-41.

SCHUBE T. 1925. Ergebnisse der Durchforschung der schlesischen Gefässpflanzenwelt im Jahre 1919-1924. Jahres-Ber. Schles. Ges. Vaterl. Cultur 97: 75-81.

SCHUBE T. 1926. Ergebnisse der Durchforschung der schlesischen Gefässpflanzenwelt im Jahre 1925. Jahres-Ber. Schles. Ges. Vaterl. Cultur 98: 9-15.

SCHUBE T. 1928. Ergebnisse der Durchforschung der schlesischen Gefässpflanzenwelt im Jahre 1927. Jahres-Ber. Schles. Ges. Vaterl. Cultur 100: 30-37.

SCHUBE T. 1929. Ergebnisse der Durchforschung der schlesischen Gefässpflanzenwelt im Jahre 1928. Jahres-Ber. Schles. Ges. Vaterl. Cultur 101: 88-96.

SENDEK A. 1966. Materiały florystyczne z północnej części Opolszczyzny za rok 1965. Opol. Tow. Przyj. Nauk, Zesz. Przyr. 6: 9-17. (in Polish with English summary)

SENDEK A. 1970. Materiały do rozmieszczenia roślin naczyniowych na Śląsku zebrane w roku 1967. Opol. Tow. Przyj. Nauk, Zesz. Przyr. 10: 23-36. (in Polish with English summary)
SOWA R. 1968. Niektóre nowe i bardziej interesujące gatunki we florze synantropijnej regionu łódzkiego, cz. II. Zesz. Nauk. Uniw. Łódz. Ser. 2, 28: 31-47. (in Polish with French summary)

SOWA R. 1969. Niektóre nowe i bardziej interesujące gatunki we florze synantropijnej regionu łódzkiego, cz. II. Zesz. Nauk. Uniw. Łódz. Ser. 2, 31: 39-55. (in Polish with French summary)

SOWA R. 1974. Wykaz gatunków flory synantropijnej Łodzi oraz zarys ich analizy geograficzno-historycznej. Zesz. Nauk. Uniw. Łódz. Ser. 2, 54: 11-26. (in Polish)

STACE C.A. 1981. Generic and infrageneric nomenclature of annual Poaceae: Poeae related to Vulpia and Desmazeria. Nord. J. Bot. 1: 17-26.

STACE C.A., AUQUIER P. 1978. Taxonomy and variation of Vulpia ciliata Dumort. Bot. J. Linn. Soc. 77: 107-112.

STACE C.A., COTTON R. 1974. Hybrids between Festuca rubra L. sensu lato and Vulpia membranacea (L.) Dumort. Watsonia 10: 119-138.

STACE C.A., COTTON R. 1980. Vulpia. In: T.G. Tutin, V.H. Heywood, N.A. Burges, D.M. Moore, D.H. Valentine, S.M. Walters, D.A. Webb (eds), Flora Europaea. 5. Alismataceae to Orchidaceae (Monocotyledones). Cambridge Univ. Press, Cambridge, pp. 154-156.

STOJANOWSKA W. 1973. Flora kamieniołomów Dolnego Śląska. Acta Univ. Wratisl. 198 Pr. Bot. 17: 35-54. (in Polish with Germany summary)

SZAFER W. 1919. Rodzina: Gramineae, Trawy. In: M. Raciborski, W. Szafer, Flora polska. Rośliny naczyniowe Polski i ziem ościennych. 1. AU, Kraków, ss. 231-365. (in Polish)

SZAFER W., KULCZYŃSKI W., PAWŁOWSKI B. 1924. Rośliny polskie. Książnica-Atlas, Lwów-Warszawa, pp. 736. (in Polish)

SZAFER W., KULCZYŃSKI W., PAWŁOWSKI B. 1953. Rośliny polskie. Wyd. 2. PWN, Warszawa, pp. 1020. (in Polish)

SZOTKOWSKI P. 1971. Materiały florystyczne z Opolszczyzny zebrane za lata 1965-1967. Opol. Tow. Przyj. Nauk, Zesz. Przyr. 11: 3-18. (in Polish with English summary)

WATSON L., DALLWITZ M.J., 1992. The grass genera of the world. C.A.B. International, Wallingford, pp. 1038.

ZAJĄC A., ZAJĄC M. (eds) 2001a. Atlas rozmieszczenia roślin naczyniowych w Polsce. Nakładem Pracowni Chorologii Komputerowej Instytutu Botaniki Uniwersytetu Jagiellońskiego, Kraków, pp. 714. (in Polish with English summary)

ZAJĄC M., ZAJĄC A. 2001b. The geographical element of the native representatives of the Gramineae (Poaceae) occurring in Poland. In: L. Frey (ed.), Studies on grasses in Poland. W. Szafer Institute of Botany, Polish Academy of Sciences, Kraków, pp. 129-139.

ZAJĄC M. 1989. Flora południowej części Kotliny Oświęcimskiej i Pogórza Śląskiego. Zesz. Naukowe Uniw. Jagiell. 952 Pr. Bot. 19: 9-199. (in Polish with English summary)

ZAPAŁOWICZ H. 1906. Krytyczny przegląd roślinności Galicyi. 1. Nakładem Akademii Umiejętności, Kraków, pp. 296. (in Polish)

ŻUKOWSKI W., JACKOWIAK B. 1995. Ginące i zagrożone rośliny naczyniowe Pomorza Zachodniego i Wielkopolski. Pr. Zakł. Takson. Rośl. Uniw. A. Mickiewicza 3: 1-141. (in Polish with English summary)

ŻUKOWSKI W. 1960. Kilka interesujących gatunków synantropijnych z miasta Poznania. Przyr. Polski Zach. 4: 141-145. (in Polish) 